\title{
Chaos and Complexity Analysis of a Discrete Permanent-Magnet Synchronous Motor System
}

\author{
Jie Ran (D), Yuqin Li, and Changchun Wang \\ School of Mathematics, Zunyi Normal College, Zunyi 563000, China \\ Correspondence should be addressed to Jie Ran; ranjie93@163.com
}

Received 8 March 2018; Revised 23 July 2018; Accepted 10 September 2018; Published 2 December 2018

Academic Editor: Sarangapani Jagannathan

Copyright ( 2018 Jie Ran et al. This is an open access article distributed under the Creative Commons Attribution License, which permits unrestricted use, distribution, and reproduction in any medium, provided the original work is properly cited.

\begin{abstract}
In this paper, a spectral entropy algorithm has been used successfully for complexity measure of a discrete permanent-magnet synchronous motor (PMSM) system. Firstly, the discrete PMSM system is achieved using the forward Euler scheme. Secondly, by adopting the bifurcation diagram, phase portraits, 0-1 test, and largest Lyapunov exponent, the chaotic dynamics of the discrete PMSM system are analyzed. The complexity of the discrete PMSM system is discussed by employing the spectral entropy algorithm. It shows that the spectral entropy complexity analysis is an efficient tool to study chaotic dynamics. Finally, we illustrate this result through numerical experiments.
\end{abstract}

\section{Introduction}

Due to its potential applications in the fields of secure communication and information encryption, chaos as a hot topic has been widely studied for nearly three decades, especially the chaotic dynamics and complexity analysis of chaotic sequences.

At present, the complexity measure of chaotic sequences can be analyzed by behavioral complexity and structure complexity. As we have known, there are several methods of measuring complexity of chaotic sequences, including the Lempl-Ziv algorithm [1, 2], ApEn algorithm [3-5], FuzzyEn algorithm [6, 7], LMC algorithm [8-10], SymEn algorithm [11], sample entropy algorithm [5], permutation entropy (PE) algorithm [12], entropy algorithm [13-16], SCM algorithm [16-18], and $\mathrm{C}_{0}$ algorithm [15, 19-22], which are widely used to analyze chaotic sequences. We should point out that the spectral entropy algorithm is based on the power spectrum of the chaotic signal and describes the irregularity of the signal spectrum, and the computation cost of this method is low [16]. Therefore, the entropy algorithm is an efficient tool to deal with complexity of chaotic sequences.
In recent years, a discrete chaotic system, which is obtained by the forward Euler scheme for its continuous system, is widely studied. A natural question to follow is whether these discrete chaotic systems are more complicated than its continuous systems or not? How to select the system parameters and step size to get more complex dynamics? Therefore, it is worthwhile to take the fluctuation of step size into full account for the dynamic problem of a chaotic system. To our knowledge, the relevant researches of these questions keep open, and these topics deserve further investigation.

Based on the above questions, the major task of this paper is to deal with complexity of chaotic sequences by means of the spectral entropy algorithm. As a result, a discrete permanent-magnet synchronous motor (PMSM) system, which is obtained by a continuous PMSM system, is considered. The PMSM system is a kind of highefficient and high-powered motor, which has been widely used in the industry [23]. The chaotic dynamics of the continuous PMSM system becomes one of most active research areas. Many important research works on the PMSM system are found in [24-27] and the references cited therein. 


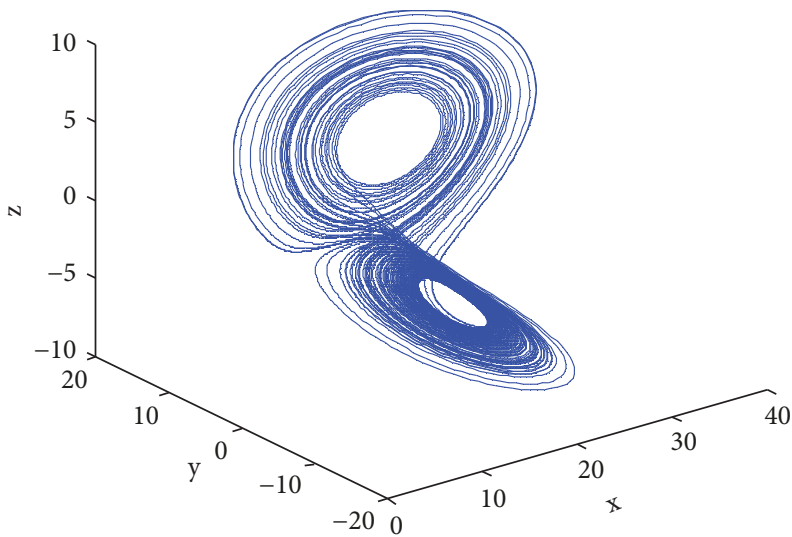

(a)

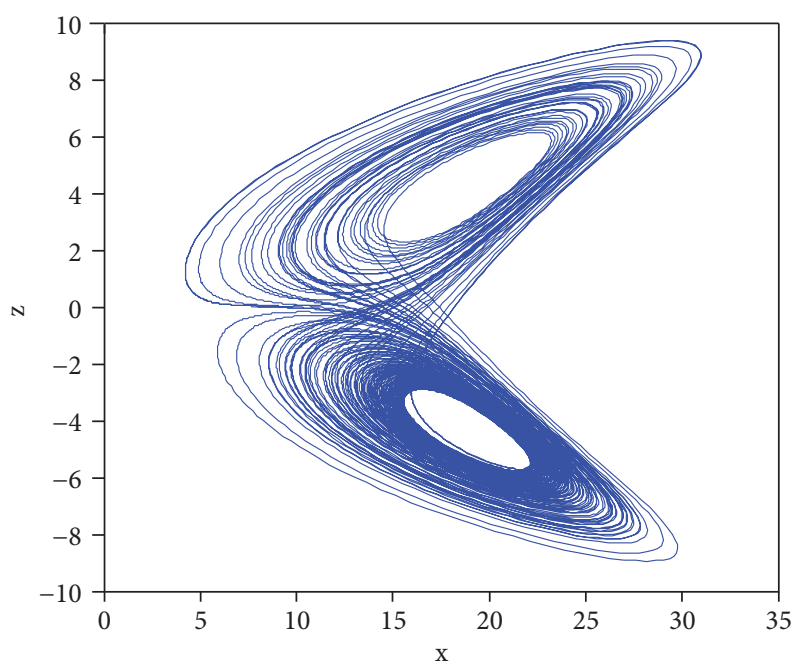

(c)

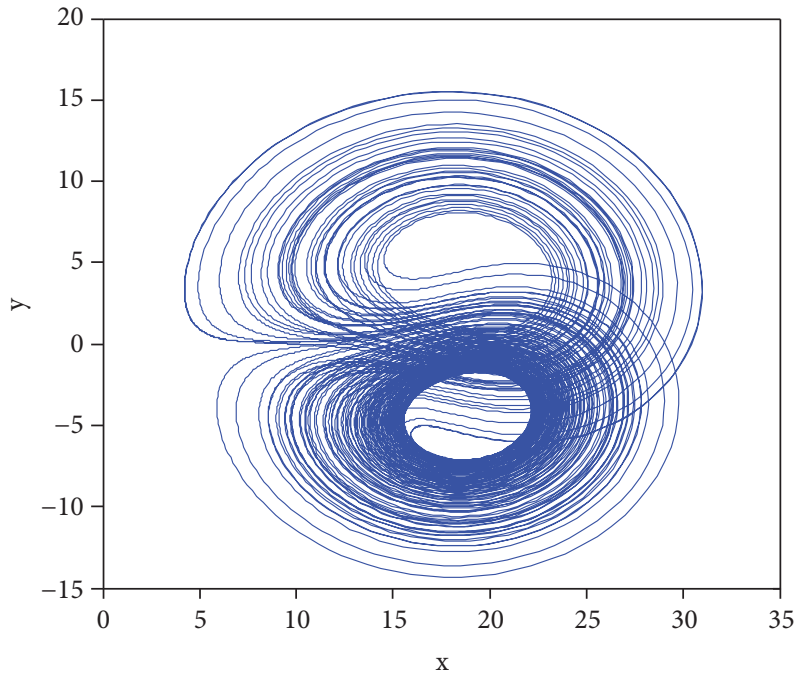

(b)

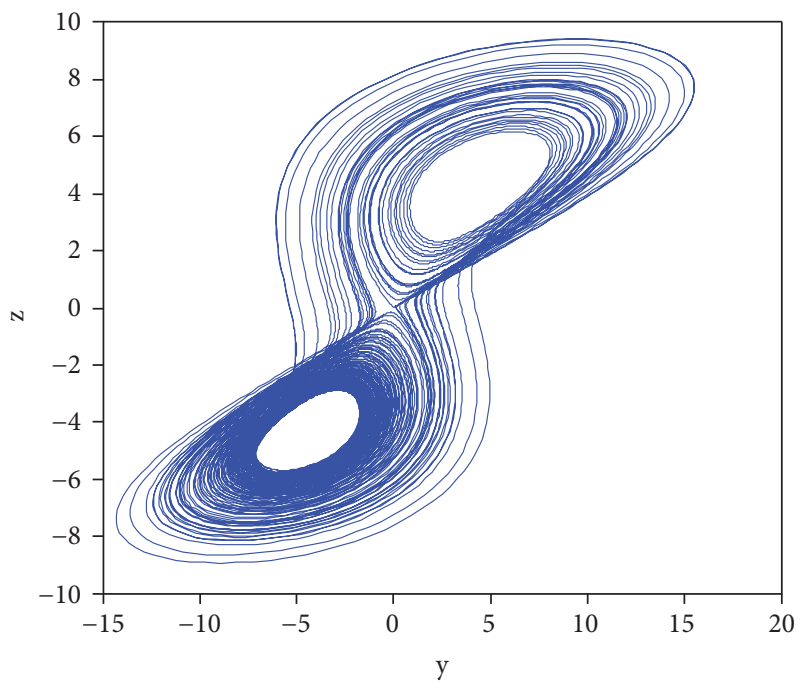

(d)

Figure 1: The 3D and 2D projections of system (1) with $(a, b)=(28,3)$ in the (a) $x-y-z$ space, (b) $x-y$ plane, (c) $x-z$ plane, and (d) $y-z$ plane, respectively.

This paper is organized as follows. In Section 2, the chaotic dynamics of the discrete PMSM system, which is obtained by using the forward Euler scheme, is investigated by means of the bifurcation diagram, phase portraits, $0-1$ test, and largest Lyapunov exponent. In Section 3, the spectral entropy algorithm is described, and the complexity of chaotic sequences in the discrete PMSM system is calculated. Finally, conclusions and future direction are presented in Section 4.

\section{Dynamics of the Three-Dimensional Discrete PMSM System}

2.1. The Three-Dimensional Discrete PMSM System. This section illustrates the application of the forward Euler method to a continuous PMSM system.
Recently, the following three-dimensional continuous PMSM system [23-27] is proposed.

$$
\left\{\begin{array}{l}
\frac{d x}{d t}=-x+y z \\
\frac{d y}{d t}=-y-x z+a z, \\
\frac{d z}{d t}=b(y-z),
\end{array}\right.
$$

where $a$ and $b$ are positive parameters. When $a=28$ and $b=3$, the PMSM system is chaotic. Its typical butterfly chaotic attractor is shown in Figure 1. When the forward Euler is employed, we obtain the following three-dimensional discrete PMSM system: 


$$
\left\{\begin{array}{l}
x(n+1)=x(n)+\delta(-x(n)+y(n) z(n)) \\
y(n+1)=y(n)+\delta(-y(n)-x(n) z(n)+a z(n)) \\
z(n+1)=z(n)+\delta(b(y(n)-z(n)))
\end{array}\right.
$$

where $a$ and $b$ are positive parameters and $\delta>0$ is the step size.

2.2. Fixed Points and Their Stability. The fixed points $O_{1}(0,0,0), O_{2}(-1+a, \sqrt{-1+a}, \sqrt{-1+a})$, and $O_{3}(-1+a$, $-\sqrt{-1+a},-\sqrt{-1+a})$ of system (2) are obtained by solving the equations:

$$
\left\{\begin{array}{l}
-x(n)+y(n) z(n)=0 \\
-y(n)-x(n) z(n)+a z(n)=0, \\
b(y(n)-z(n))=0 .
\end{array}\right.
$$

In order to study the stability of the fixed point $O_{1}$, we calculate the Jacobian matrix of system (2) at $O_{1}$ as

$$
J_{O_{1}}=\left[\begin{array}{ccc}
1-\delta & 0 & 0 \\
0 & 1-\delta & a \delta \\
0 & b \delta & 1-b \delta
\end{array}\right]
$$

and its characteristic polynomial equation is

$$
\lambda^{3}+\alpha \lambda^{2}+\beta \lambda+\gamma=0
$$

where $\alpha=2 \delta+b \delta-3, \beta=2 b \delta^{2}+\delta^{2}-a b \delta^{2}-4 \delta-2 b \delta+3$, and $\gamma=b \delta^{3}-a b \delta^{3}+a b \delta^{2}-2 b \delta^{2}-\delta^{2}+2 \delta+b \delta-1$. According to the Schur-Cohn criterion [28], we derive the stability conditions of $O_{1}$ as follows: (a) $|\alpha+\gamma|<1+\beta$ and (b) $|\beta-\alpha \gamma|<1-\gamma^{2}$. As a result, by taking $b=3$ and $\delta=0.01$, the stability condition of $O_{1}$ is $0<a<1$. Here, we only consider stability of the fixed point $O_{1}$, and the stability of the fixed points $\mathrm{O}_{2}$ and $\mathrm{O}_{3}$ can be studied in a similar manner as for $O_{1}$.

2.3. Chaos in the Three-Dimensional Discrete PMSM System. In this section, by means of the phase portraits, bifurcation diagram, 0-1 test and largest Lyapunov exponent, the dynamics of system (2) is researched.

In particular, variations of the parameter $a$ are considered by keeping $b=3$ and $\delta=0.02$. The largest Lyapunov exponent diagram of system (2) is obtained using the Jacobi matrix method as shown in Figure 2. Letting $\delta=0.02$ and fixing $b=3$, the bifurcation diagram of system (2) in Figure 3 is obtained for $a$ in the range $(0,30)$. From Figures 2 and 3, of particular interest is that it has a chaotic attractor with one positive Lyapunov exponent in a large range of parameters. As a result, starting with $a=8$, a stable solution is obtained, and the corresponding phase diagram is given in Figure 4(a). By increasing the value of $a$, a two-scroll chaotic attractor is obtained for $a=28$ as shown in Figure 4(b). By adopting 0-1 test [29], the corresponding trajectories in the $(p-s)$ plane are shown in Figures 5(a)

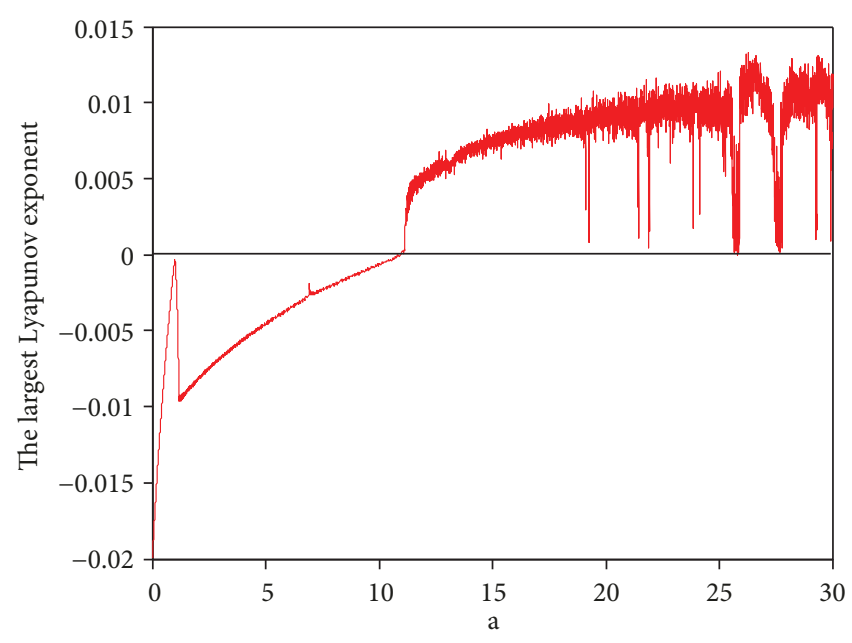

FIgURE 2: The largest Lyapunov exponent of system (2) with $(b, \delta)=(3,0.02)$ and $a \in(0,30)$.

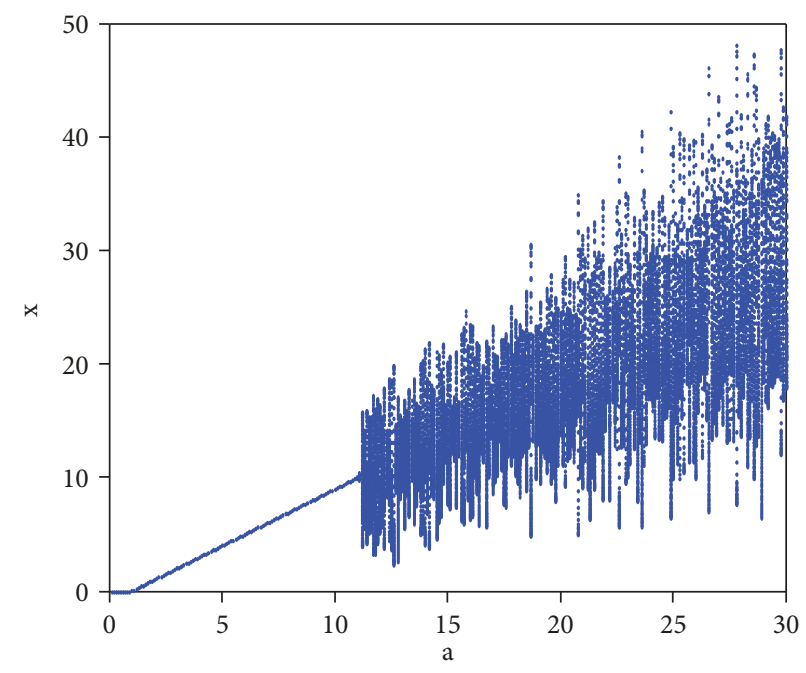

FIgURE 3: The bifurcation diagram of system (2) with $(b, \delta)=$ $(3,0.02)$ and $a \in(0,30)$.

and 5(b). Here, $p$ and $s$ are two real valued functions, respectively,

$$
\begin{aligned}
& p(n)=\sum_{j=1}^{n} \phi(j) \cos (\theta(j)), \\
& s(n)=\sum_{j=1}^{n} \phi(j) \sin (\theta(j)),
\end{aligned}
$$

where $\theta(j)=j c+\sum_{i=1}^{j} \phi(i), j=1,2,3 \cdots, n$, and $c$ is a random number in the range $[\pi / 5,4 \pi / 5]$. Also note that bounded trajectories in the $(p-s)$ plane correspond to regular dynamics, whereas Brownianlike (unbounded) trajectories correspond to chaotic dynamics [29]. See Gottwald and Melbourne [29] for details. 


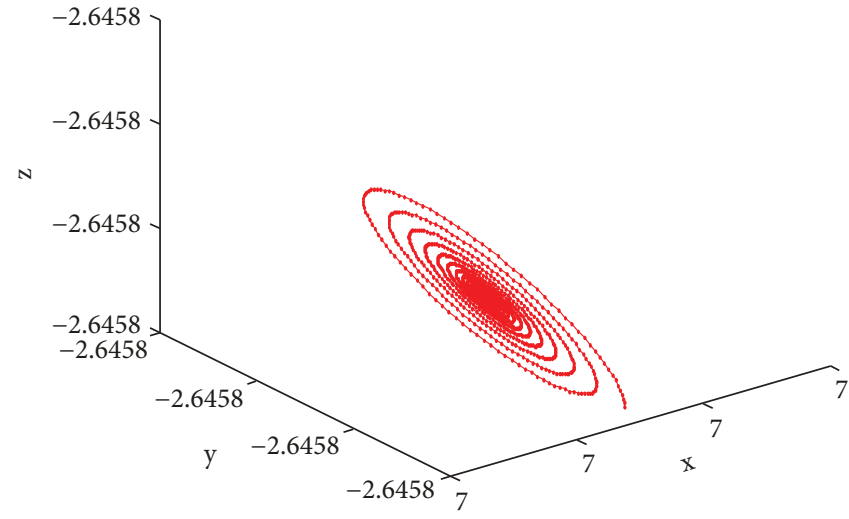

(a)

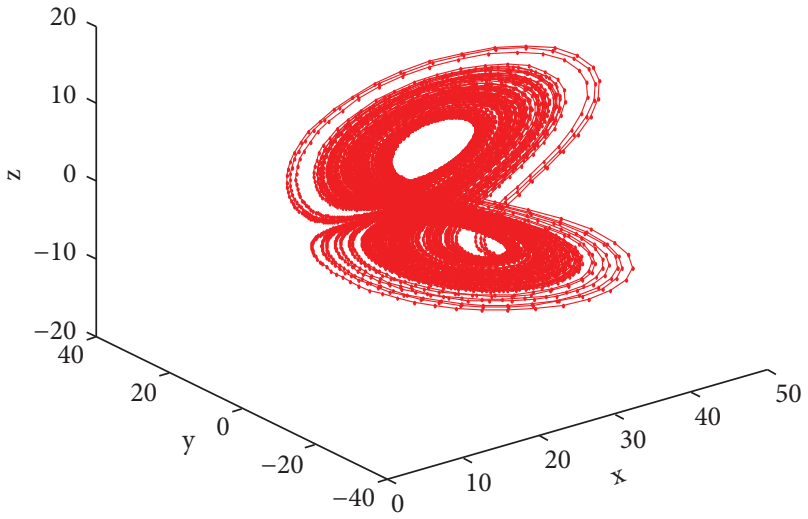

(b)

FIgUre 4: The phase diagrams of system (2) with $(b, \delta)=(3,0.02):$ (a) $a=8$ and (b) $a=28$.

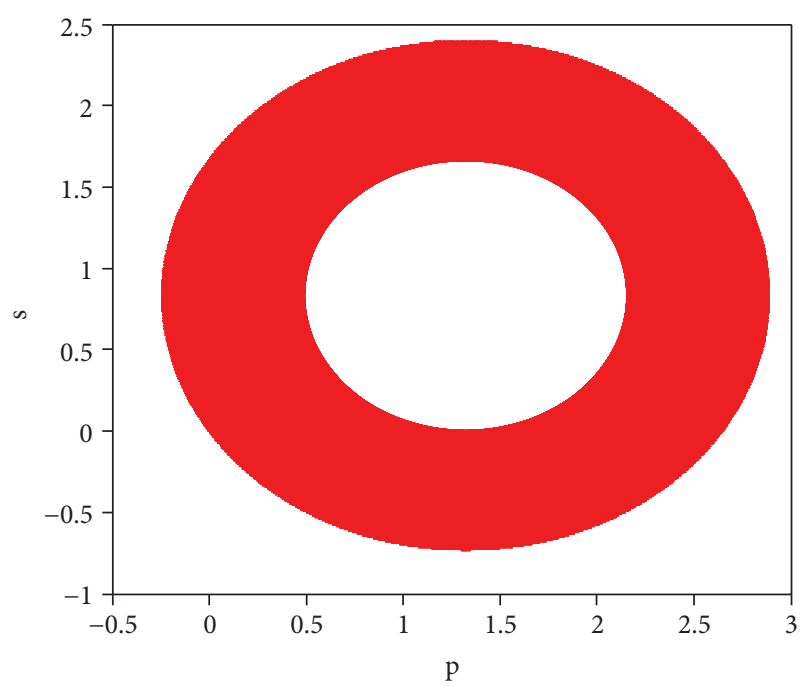

(a)

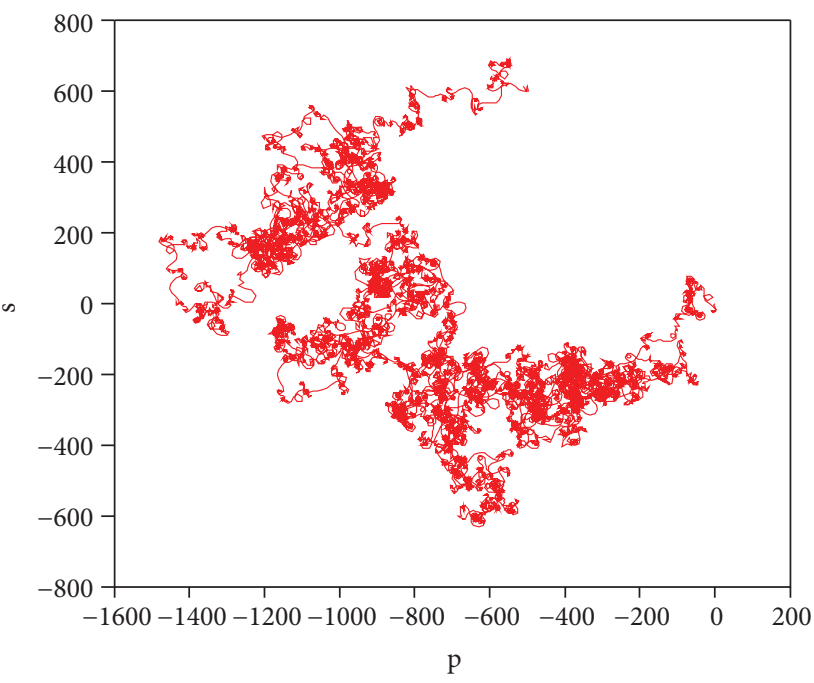

(b)

FIgURe 5: The dynamics of the translation components $(p-s)$ of system (2) with (a) $a=8$ and (b) $a=28$.

From Figures 2-4, it is clear that the stable, periodic solutions and ensuing transition to chaos are observed.

\section{Complexity Analysis}

In this section, the complexity of system (2) is discussed by employing the spectral entropy algorithm.

The spectral entropy algorithm is a method that deals with the complexity of a time series. The spectral entropy reflects the disorder in the Fourier transformation domain, if a flatter spectrum has a larger value of spectral entropy, which shows a higher complexity of the time series [16]. Consider a set of discrete data $\{\phi(n), n=0,1,2, \cdots, N-1\}$ with a length of $N$, representing a one-dimensional observable data set. According to discrete Fourier transformation approach, the spectral entropy algorithm [13-16] is briefly described as follows.
Step 1. Data processing. In order to better reflect the energy information of the data $\phi(n)$, transform the data $\phi(n)$ as follows:

$$
\phi^{\prime}(n)=\phi(n)-\bar{\phi}
$$

where $\bar{\phi}=(1 / N) \sum_{n=0}^{N-1} \phi(n)$.

Step 2. Discrete Fourier transformation. Taking the discrete Fourier transform for the data $\phi^{\prime}(n)$ and its corresponding discrete Fourier transform is defined as

$$
\Phi(k)=\sum_{n=0}^{N-1} \phi^{\prime}(n) e^{-j(2 \pi / N) n k}=\sum_{n=0}^{N-1} \phi^{\prime}(n) W_{N}^{n k}
$$

where $k=0,1, \ldots N-1$ and $j=\sqrt{-1}$ is the unit imaginary. 


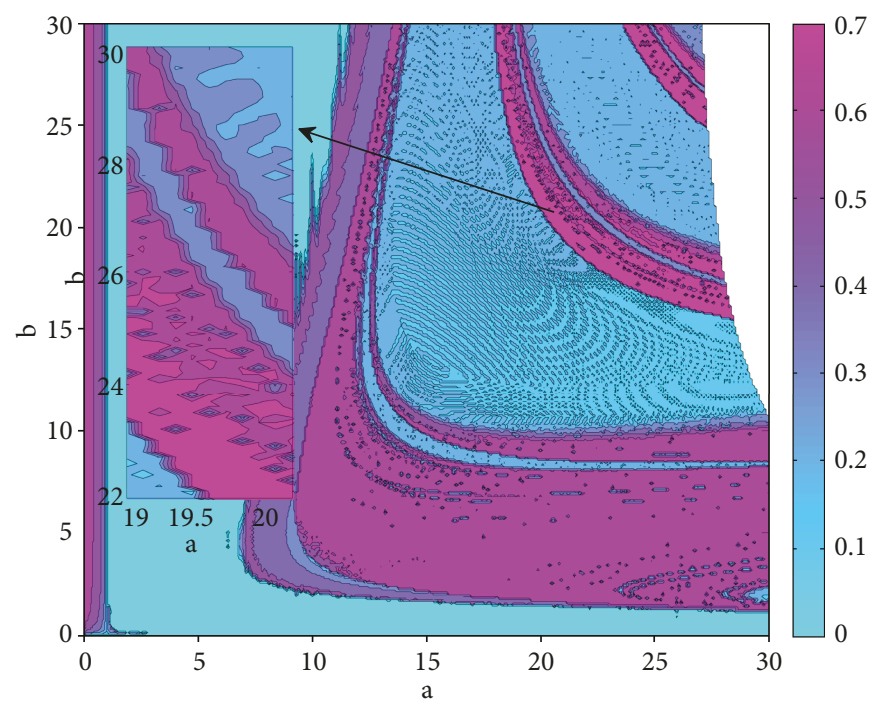

FIGURE 6: The spectral entropy complexity of system (2) with $\delta=0.02$ in the $(a-b)$ parameter plane.

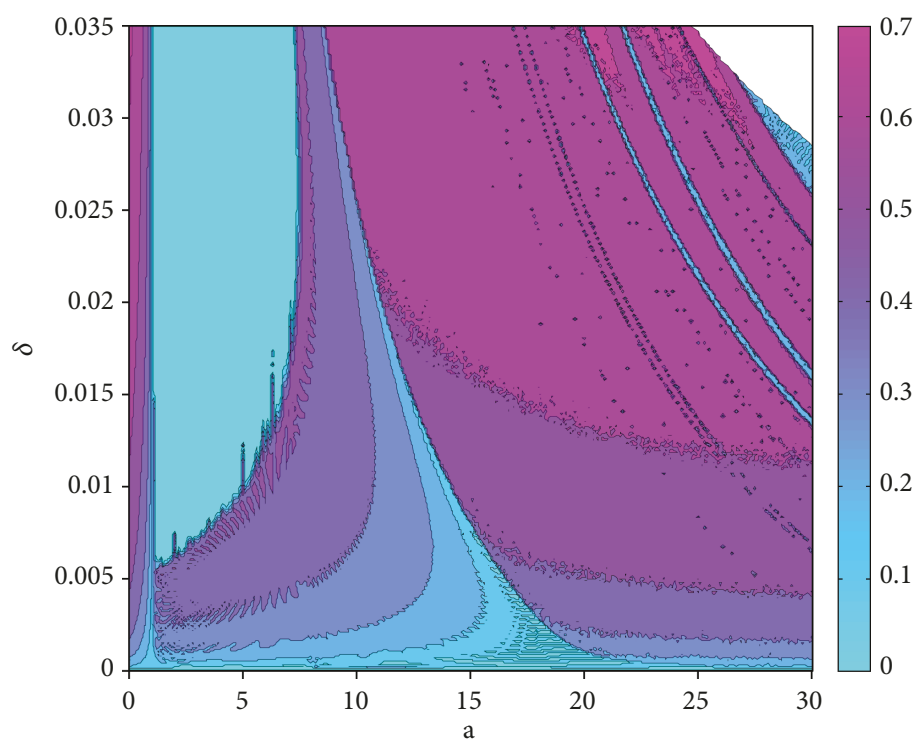

FIgURE 7: The spectral entropy complexity of system (2) with $b=3$ in the $(a-\delta)$ parameter plane.

Step 3. Calculating relative power spectrum. If the power of a discrete power spectrum with the $k$ th frequency is $|\Phi(k)|^{2}$, then the "probability" of this frequency is defined as

$$
P_{k}=\frac{(1 / N)|\Phi(k)|^{2}}{(1 / N) \sum_{k=0}^{N / 2-1}|\Phi(k)|^{2}}=\frac{|\Phi(k)|^{2}}{\sum_{k=0}^{N / 2-1}|\Phi(k)|^{2}} .
$$

Step 4. Spectral entropy is calculated. Applying the $P_{k}$, the spectral entropy is defined by

$$
S E=\frac{1}{\ln (N / 2)}\left(\sum_{k=0}^{N / 2-1}\left|P_{k} \ln P_{k}\right|\right) .
$$

Based on the above four steps, the spectral entropy of a given time series with length $N$ is calculated, without choosing any other parameters [15].

Here, we choose parameter planes $(a, b),(a, \delta)$, and $(b, \delta)$ to calculate its complexity, for example, to see how the complexity changes as the parameters $a, b$, and $\delta$ increase (or decrease). As a result, the length of data $\{\phi(n), n=1,2 \cdots\}$ for spectral entropy is $4 \times 10^{4}$ after removing the first $1 \times 10^{4}$ points of data [15]. By taking $\delta=0.02$, the spectral entropy complexity of $x$ series of system (2) is calculated and illustrated in Figure 6.

From Figure 6, it can be seen that the parameter $b$ has a larger range of choices when parameter $a$ is smaller. For example, the parameter $a$ is selected in the range $(0,1)$; the system (2) maintains a high complexity for parameter 


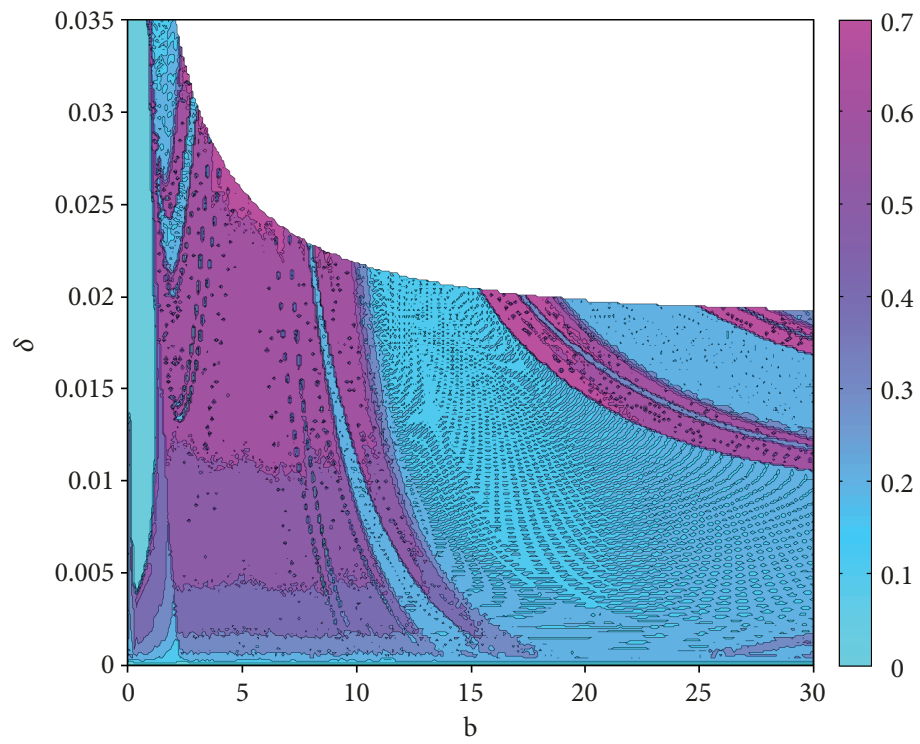

FIGURE 8: The spectral entropy complexity of system (2) with $a=28$ in the $(b-\delta)$ parameter plane.

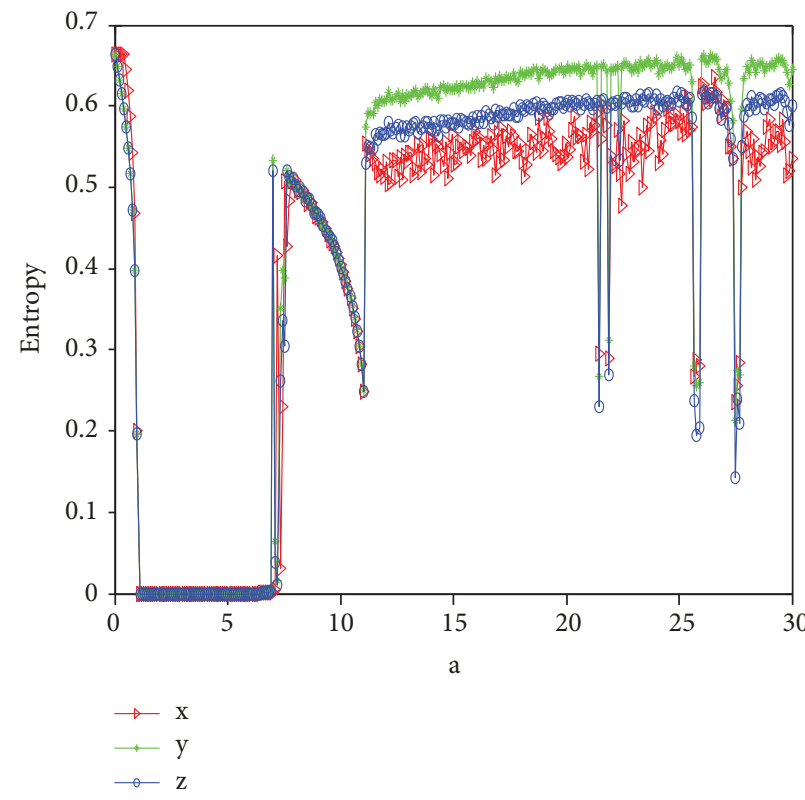

(a)

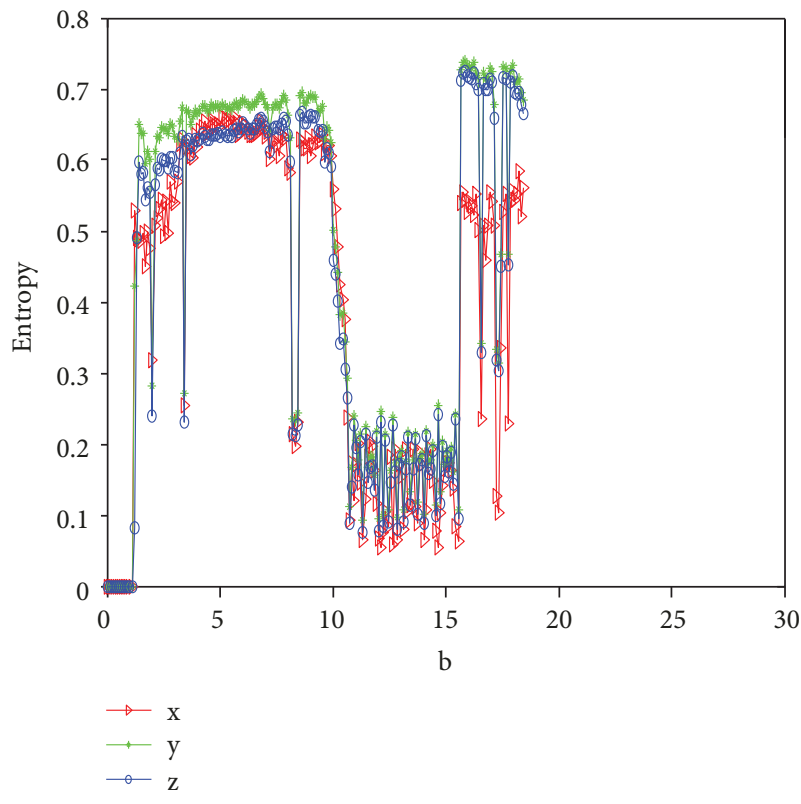

(b)

FiguRE 9: The spectral entropy complexity of system (2) (a) versus $a \in(0,30)$ with $(b, \delta)=(3,0.02)$; (b) versus $b \in(0,30)$ with $(a, \delta)=$ $(28,0.02)$.

$b \in(0,30)$. When the parameters $a$ and $b$ are selected in the two light-colored areas in the upper right, the system (2) maintains the largest complexity. Hence, for this chaotic system (2), the complexity depends on parameters $a$ and $b$ sensitively.

Next, we calculate the complexity of system (2), when the step size $\delta$ is selected in the range $(0,0.035)$. By taking $b=3$, the spectral entropy complexity in Figure 7 is obtained for parameters $a$ and $\delta$ in the range $(0,30)$ and $(0,0.035)$, respectively.
From Figure 7, it can be seen that the largest value of spectral entropy complexity of system (2) fluctuates within a small range for parameters $a$ and $\delta$. Similarly, by taking $a=28$, the spectral entropy complexity in Figure 8 is obtained for parameters $b$ and $\delta$ in the range $(0,30)$ and $(0,0.035)$, respectively. According to Figure 8, it can be seen that the largest value of spectral entropy complexity of system (2) is 0.7 . For parameters $b$ and $\delta$, they are roughly in the range of $(3,10)$ and $(0.015$, $0.0 .025)$, respectively. 


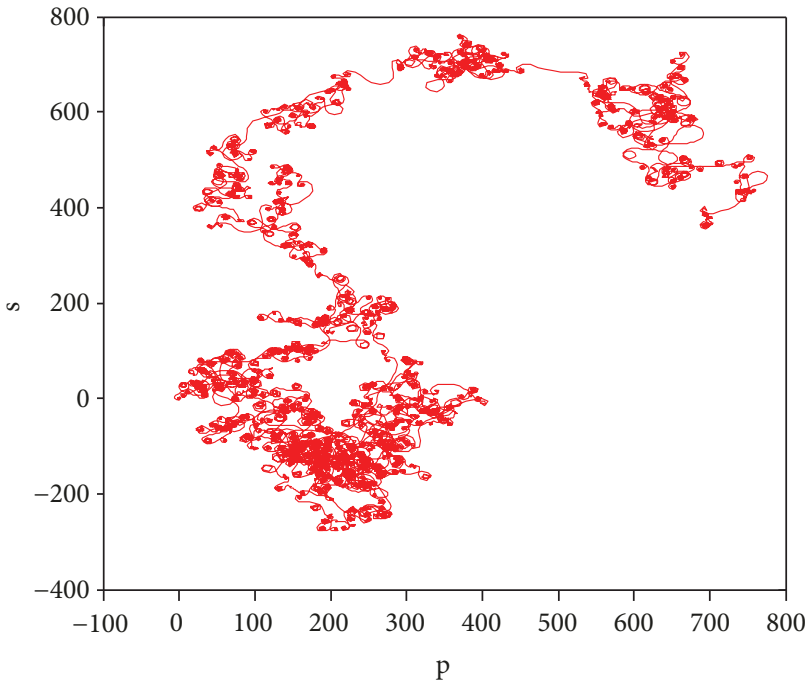

FIgURE 10: The dynamics of the translation components $(p-s)$ of system (2) with $a=15, b=3$, and $\delta=0.02$.

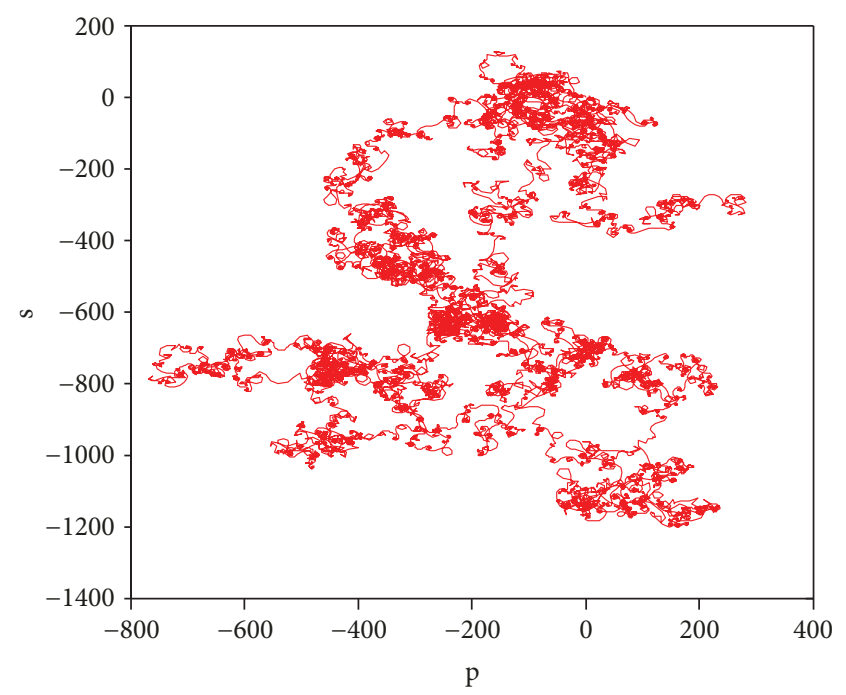

FIGURE 11: The dynamics of the translation components $(p-s)$ of system (2) with $a=28, b=7$, and $\delta=0.02$.

Based on the discussion above, we found that spectral entropy complexity analysis is a convenient method to choose parameters for a discrete system.

In the following discussion, two of the parameters are fixed and the spectral entropy complexity is calculated. By taking $b=3$ and $\delta=0.02$, the spectral entropy complexity in Figure 9(a) is obtained for parameter $a$ in the range $(0,30)$. Similarly, fixing $a=28$ and $\delta=0.02$, the spectral entropy complexity is calculated for parameter $b$ in the range $(0,30)$ as shown in Figure $9(\mathrm{~b})$. Combined with Figure 9, we find that the spectral entropy complexity calculation is similar to that of the Lyapunov exponent, which reflects the complexity of chaos. In Figure 9(a), letting $a=15, b=3$, and $\delta=0.02$, the $0-1$ test is obtained as shown in Figure 10. In Figure 9(b), letting $a=28, b=7$, and $\delta=0.02$, the $0-1$ test is obtained as shown in Figure 11 .

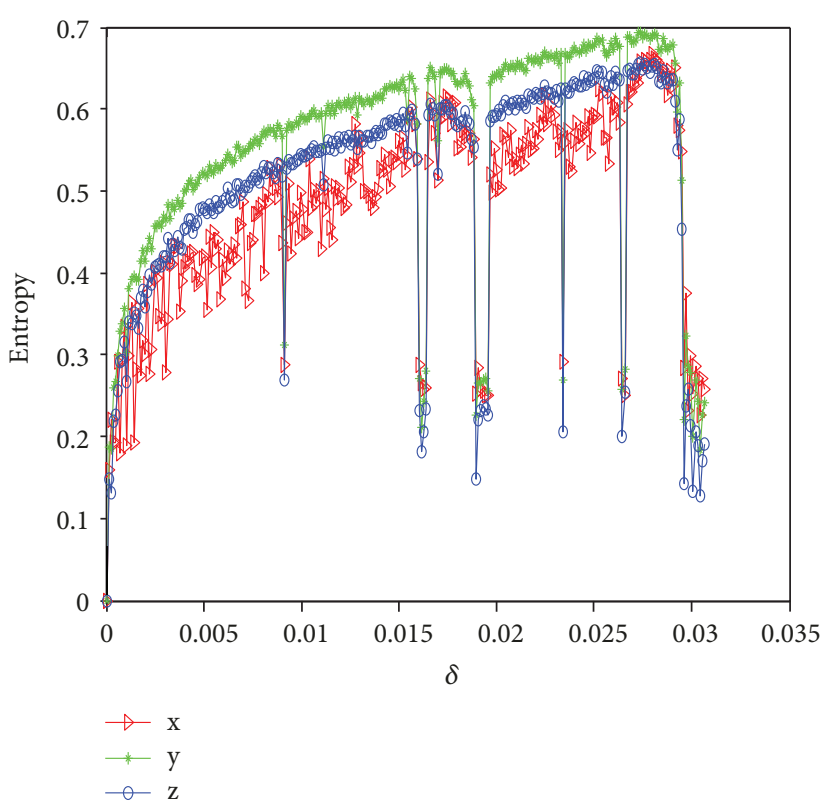

FIgURE 12: The spectral entropy complexity of system (2) versus step size $\delta \in(0,0.035)$ with $(a, b)=(28,3)$.

In addition, it is worth noting that the spectral entropy complexity of system (2) increases with the increasing step size $\delta$. In particular, variations of the step size $\delta$ is considered by keeping $a=28$ and $b=3$. The spectral entropy complexity is shown in Figure 12.

\section{Conclusions}

In this paper, by means of the largest Lyapunov exponent, phase diagram, bifurcation diagram, 0-1 test, and spectral entropy algorithm, we investigated the chaotic dynamics and complexity of the discrete PMSM system obtained by the forward Euler scheme. The conclusions are drawn as follows.

The discrete PMSM system contains rich dynamical behaviors. The two-scroll stronger chaotic attractor is observed with the increasing parameter $a$. Moreover, the discrete PMSM system is chaotic in large range for parameter $a$. Furthermore, by calculating the chaos complexity of discrete PMSM system, we found that it has a high complexity. Therefore, the discrete PMSM system has higher application value in the real-word applications. For example, this system can be considered for chaotic encryption.

We also found an interesting phenomenon that the spectral entropy complexity of discrete PMSM system increases with increasing step size $\delta$. Thus, the spectral entropy complexity analysis is a convenient method to choose step size. In fact, it also provides a reference for Euler method to study the chaotic dynamics of continuous dynamical system.

It is expected that the results enrich our knowledge of the dynamics of discrete systems, which is obtained by the forward Euler scheme. Furthermore, it suggests that the results are beneficial to researchers who explore other dynamical behaviors. 


\section{Data Availability}

No additional data are available for the paper.

\section{Conflicts of Interest}

The authors declare that there is no conflict of interests regarding the publication of this paper.

\section{Acknowledgments}

This work is supported by the Guizhou Science and Technology Cooperation Plan (Qian Ke He LH zi [2016]7032 and Qian Ke He LH zi [2016]7031) and Youth Science and Technology Talents Growth Projects of Department of Education of Guizhou Province (no. 12847, Qian Jiao He KY Zi [2018]315, [2018]313, [2017]256, and [2017]251 and Qian Ke He LH Zi [2017]7072).

\section{References}

[1] A. Lempel and J. Ziv, "On the complexity of finite sequences," IEEE Transactions on Information Theory, vol. 22, no. 1, pp. 75-81, 1976.

[2] L. Nian-sheng, "Pseudo-randomness and complexity of binary sequences generated by the chaotic system," Communications in Nonlinear Science and Numerical Simulation, vol. 16, no. 2, pp. 761-768, 2011.

[3] K. H. Sun, G. Q. Tan, and L. Y. Sheng, "The complexity analysis of TD-ERCS discrete chaotic pseudo-random sequences," Acta Physica Sinica, vol. 57, no. 2, pp. 3359-3366, 2008.

[4] S. Pincus, "Approximate entropy (ApEn) as a complexity measure," Chaos, vol. 5, no. 1, pp. 110-117, 1995.

[5] J. S. Richman and J. R. Moorman, "Physiological time-series analysis using approximate entropy and sample entropy," American Journal of Physiology-Heart and Circulatory Physiology, vol. 278, no. 6, pp. H2039-H2049, 2000.

[6] W. Chen, Z. Wang, H. Xie, and W. Yu, "Characterization of surface EMG signal based on fuzzy entropy," IEEE Transactions on Neural Systems and Rehabilitation Engineering, vol. 15, no. 2, pp. 266-272, 2007.

[7] X. J. Chen, Z. Li, B. M. Bai, W. Pan, and Q. H. Chen, "A new complexity metric of chaotic pseudorandom sequences based on fuzzy entropy," Journal of Electronics \& Information Technology, vol. 33, no. 5, pp. 1198-1203, 2011.

[8] K. H. Sun, S. B. He, and L. Y. Sheng, "Complexity analysis of chaotic sequence based on the intensive statistical complexity algorithm," Acta Physica Sinica, vol. 60, no. 2, pp. 90-96, 2011.

[9] F. H. Xiao, G. R. Yan, and Y. H. Han, "Information theory approach to determine embedding parameters for phase space reconstruction of chaotic time series," Acta Physica Sinica, vol. 54, no. 2, pp. 550-556, 2005.

[10] H. A. Larrondo, C. M. González, M. T. Martín, A. Plastino, and O. A. Rosso, "Intensive statistical complexity measure of pseudorandom number generators," Physica A: Statistical Mechanics and its Applications, vol. 356, no. 1, pp. 133-138, 2005.

[11] R. K. Azad, J. Subba Rao, and R. Ramaswamy, "Informationentropic analysis of chaotic time series: determination of time-delays and dynamical coupling," Chaos, Solitons \& Fractals, vol. 14, no. 4, pp. 633-641, 2002.
[12] C. Bandt and B. Pompe, "Permutation entropy: a natural complexity measure for time series," Physical Review Letters, vol. 88, no. 17, article 174102, 2002.

[13] T. Inouye, K. Shinosaki, H. Sakamoto et al., "Quantification of EEG irregularity by use of the entropy of the power spectrum," Electroencephalography and Clinical Neurophysiology, vol. 79, no. 3, pp. 204-210, 1991.

[14] K. H. Sun, S. B. He, Y. He, and L. Z. Yin, "Complexity analysis of chaotic pseudo-random sequences based on spectral entropy algorithm," Acta Physica Sinica, vol. 62, no. 1, pp. 27-34, 2013.

[15] S. He, K. Sun, and H. Wang, "Complexity analysis and DSP implementation of the fractional-order Lorenz hyperchaotic system," Entropy, vol. 17, no. 12, pp. 8299-8311, 2015.

[16] S. B. He, K. H. Sun, and C. X. Zhu, "Complexity analyses of multi-wing chaotic systems," Chinese Physics B, vol. 22, no. 5, article 050506, 2013.

[17] R. López-Ruiz, H. L. Mancini, and X. Calbet, "A statistical measure of complexity," Physics Letters A, vol. 209, no. 5-6, pp. 321-326, 1995.

[18] C. M. González, H. A. Larrondo, and O. A. Rosso, "Statistical complexity measure of pseudorandom bit generators," Physica A: Statistical Mechanics and its Applications, vol. 354, pp. 281300, 2005.

[19] K. H. Sun, S. B. He, C. X. Zhu, and Y. He, "Analysis of chaotic complexity characteristics based on $\mathrm{C}_{0}$ algorithm," Acta Electronica Sinica, vol. 41, no. 9, pp. 1765-1771, 2013.

[20] F. Chen, J. Xu, F. Gu, X. Yu, X. Meng, and Z. Qiu, "Dynamic process of information transmission complexity in human brains," Biological Cybernetics, vol. 83, no. 4, pp. 355-366, 2000.

[21] S. En-hua, C. Zhi-jie, and G. Fan-ji, "Mathematical foundation of a new complexity measure," Applied Mathematics and Mechanics, vol. 26, no. 9, pp. 1188-1196, 2005.

[22] Y. Cao, Z. Cai, E. Shen et al., "Quantitative analysis of brain optical images with $2 \mathrm{D} C_{0}$ complexity measure," Journal of Neuroscience Methods, vol. 159, no. 1, pp. 181-186, 2007.

[23] Z. Jing, C. Yu, and G. Chen, "Complex dynamics in a permanent-magnet synchronous motor model," Chaos, Solitons \& Fractals, vol. 22, no. 4, pp. 831-848, 2004.

[24] Z. Li, J. B. Park, Y. H. Joo, B. Zhang, and G. Chen, "Bifurcations and chaos in a permanent-magnet synchronous motor," IEEE Transactions on Circuits and Systems I: Fundamental Theory and Applications, vol. 49, no. 3, pp. 383-387, 2002.

[25] H. H. Choi, "Adaptive control of a chaotic permanent magnet synchronous motor," Nonlinear Dynamics, vol. 69, no. 3, pp. 1311-1322, 2012.

[26] J. Wang, W. Zhou, and X. Zhou, "Chaos control of pulsedisturbed permanent magnet synchronous motor with uncertain parameters," Transactions of the Institute of Measurement and Control, vol. 38, no. 7, pp. 876-883, 2016.

[27] F. Zhang, X. Liao, and C. Mu, "Dynamical analysis of the permanent-magnet synchronous motor chaotic system," Advances in Difference Equations, vol. 2017, no. 1, 2017.

[28] J. P. LaSalle, The Stability and Control of Discrete Processes, vol. 62 of Applied Mathematical Sciences, , Springer, 1986.

[29] G. A. Gottwald and I. Melbourne, "On the implementation of the $0-1$ test for chaos," SIAM Journal on Applied Dynamical Systems, vol. 8, no. 1, pp. 129-145, 2009. 


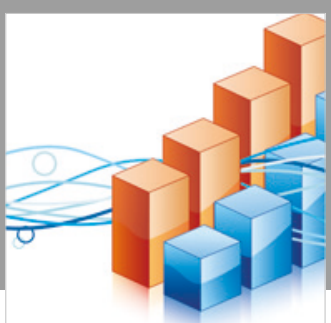

Advances in

Operations Research

\section{-n-m}
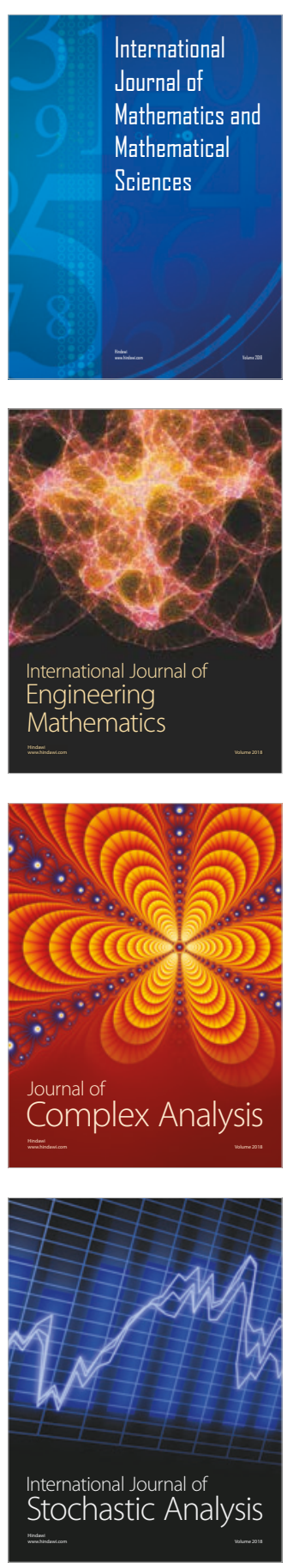
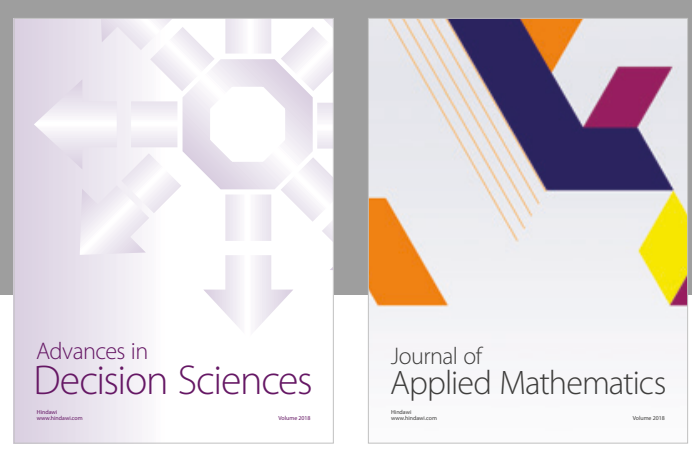

Journal of

Applied Mathematics
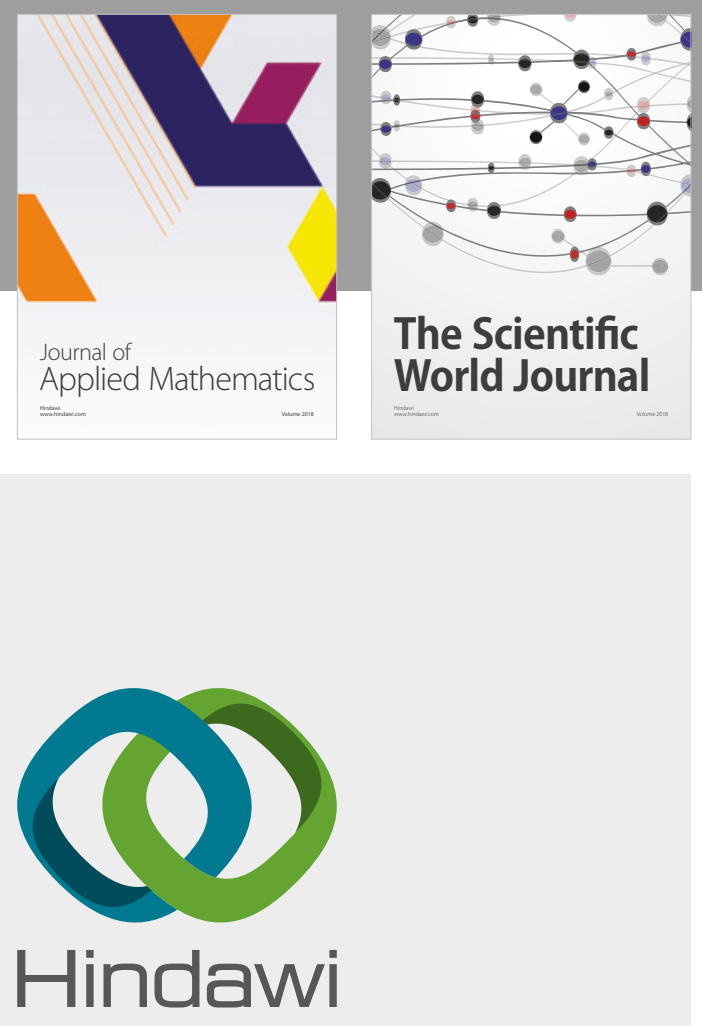

Submit your manuscripts at

www.hindawi.com

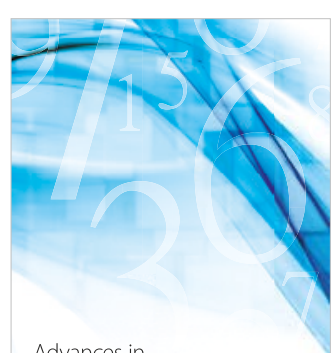

Advances in
Numerical Analysis
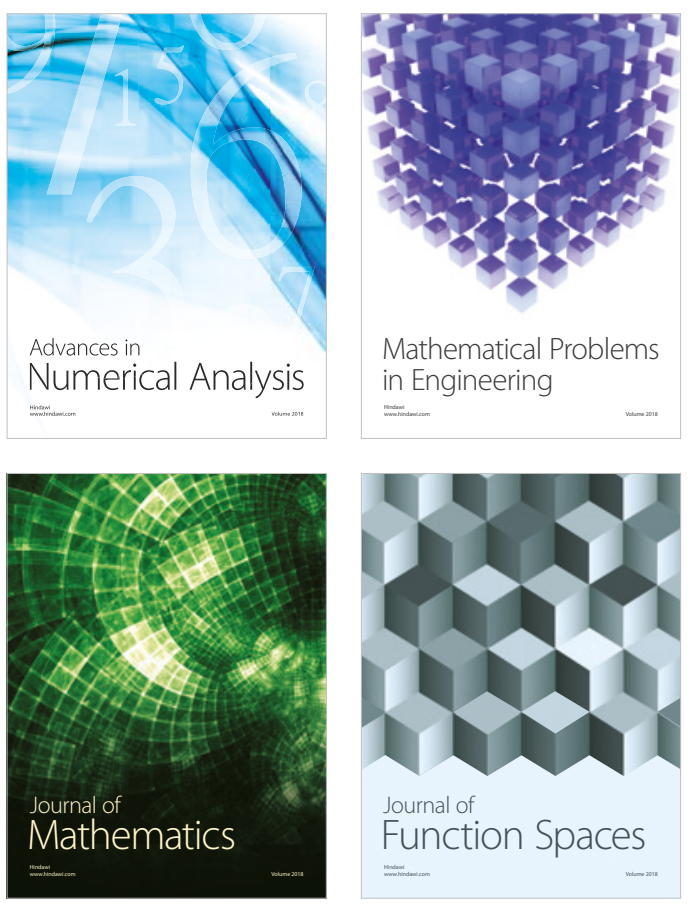

Mathematical Problems in Engineering

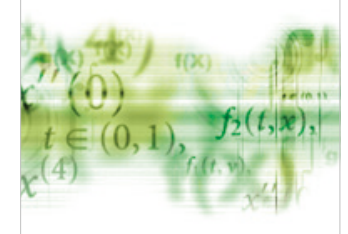

International Journal of

Differential Equations

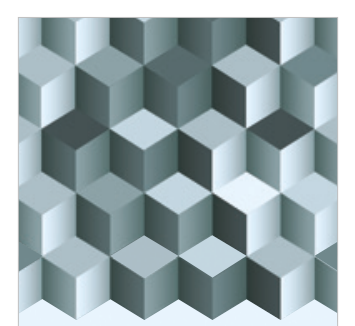

Journal of

Function Spaces
The Scientific

World Journal

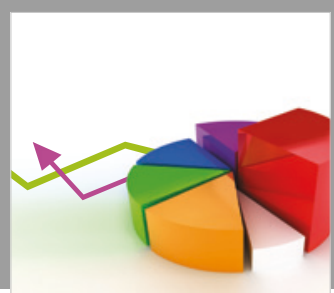

Journal of

Probability and Statistics
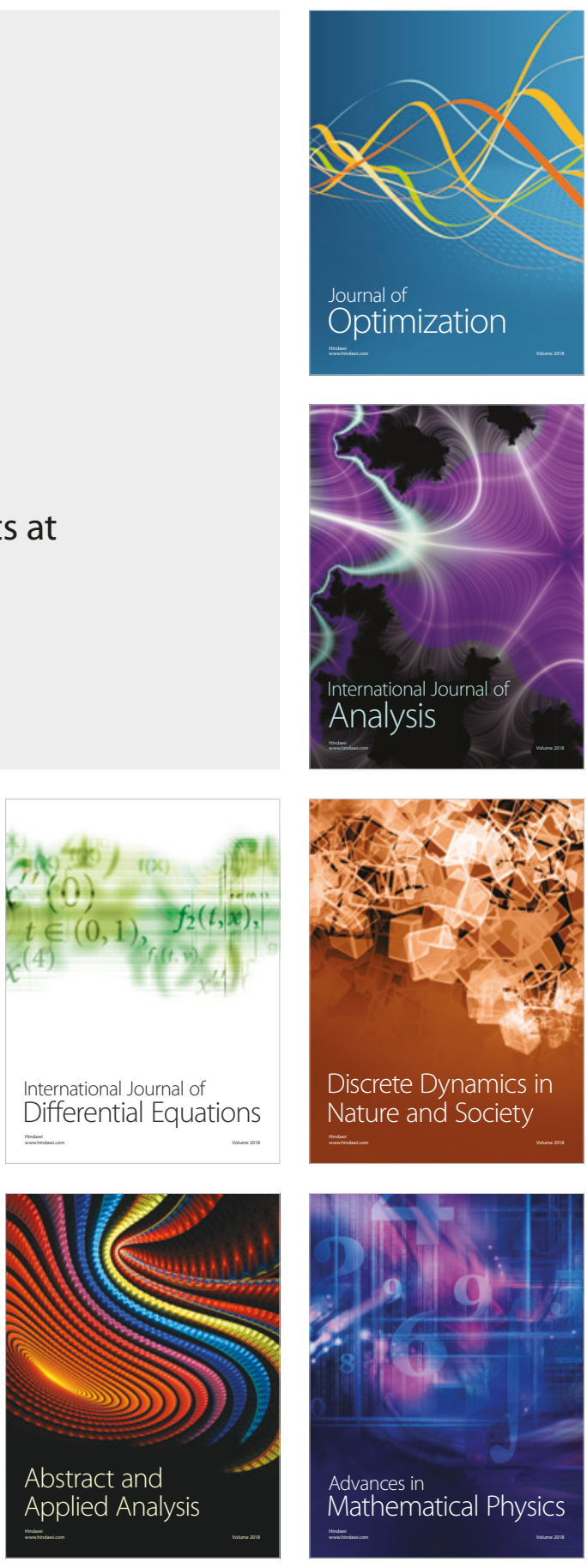